\title{
A Mimicking Human Arm with 5 DOF Controlled by LabVIEW
}

\author{
Basil Hamed
}

\begin{abstract}
In this paper, a proposed design of 5 DOF arm robot is presented; each of the arm's joints will be generated by the corresponding human arm's joints. The paper will present a full design of a robot arm that mimics the human arm motion. Also, a graphical simulator will be designed to mimic the human arm simultaneously with the mechanical robot arm. Both of the arms are controlled using LabVIEW and data acquisition card NI-PCI 6024E. For control applications, LabVIEW Real-Time provide a single software development tool that can implement the functions to PC-based control systems, thus acting as a programmable automation controller.
\end{abstract}

Index Terms-Control, Human-Machine Interface, LabVIEW, Data Acquisition, 5DOF, and Human Motion

\section{INTRODUCTION}

Among social infrastructure technologies, Robot technology is expected to play an important role in solving the world problems. One of the most important fields in the development of successful robotics systems is the Human-Machine Interaction. A control system is a collection of components working together under the direction of some machine intelligence [1]. Nowadays, many kinds of mechanical arms are used in various applications such as in semiconductor fabrications, automobile manufacturing, various industries, medical operations, transportations, educations, or even in space missions [2][3]. Robots are most useful in executing the same defined job repeatedly and in precisely the same fashion. When working on tasks that are continuously duplicated, these automated machines are able to overcome certain types of errors better than humans. In most cases, electronic circuits provide the intelligence, and electromechanical components such as sensors and motors provide the interface to the physical world and by this work it introduces intelligence to the system by computer providing via LabVIEW software with Data Acquisition Card utility. Robot arms are versatile tools found in a wide range of applications. In recent years, applications where humans and robot arms interact, have received increased attention. The case where the interaction entails the human controlling a remote robot is called robot teleoperation. The latter case requires a user interface to translate the operator commands to the robot. A large number of interfaces have been proposed on this issue in previous works, where some examples of them can be found in [4], [5], and [6]. However, most of them involve complex mechanisms or systems of sensors, that the user should be acquaint with, resulting to a feeling of discomfort for non-experts. Moreover, in most of the cases the user should be trained to map his/her action (i.e. three dimensions motion of a joystick) to the resulted motion of the robot. In this paper, a new mean of interface is proposed, according to which, the user can directly control 5 DOF a robot arm, by performing natural motions with his/her own arm, using LabVIEW software and data acquisition card. The robot-arm has 5 degrees of freedom (DOF). The shoulder is bowing and rotating, the elbow and the wrist are only bowing and the hand itself is rotating

The first and foremost factor in designing the robotic arm is the decision making of the number of DOF. This decision is mainly dependent on the task which the manipulator has to perform and the workspace it has to cover. In simple pick and place operations the suitable DOFs can make a system robust but complicated as well. The larger the DOF the more efficient and dexterous the manipulator is. But on the other hand, system complication in design and control also increases [7]. In this paper 5 DOF arm robot has been designed.

\section{Design Criteria}

The arm robot system must achieve the following design criterions:

The arm robot system should have compatible shape to be suitable for human usage.

Stability of the arm robot system is the main factor that reflects the quality of the design.

\section{SYSTEM OVERVIEW}

To achieve the design criteria, the system consists of three parts. Figure (1) shows the main parts of the system and the flow of data from input to output passing through the controller.

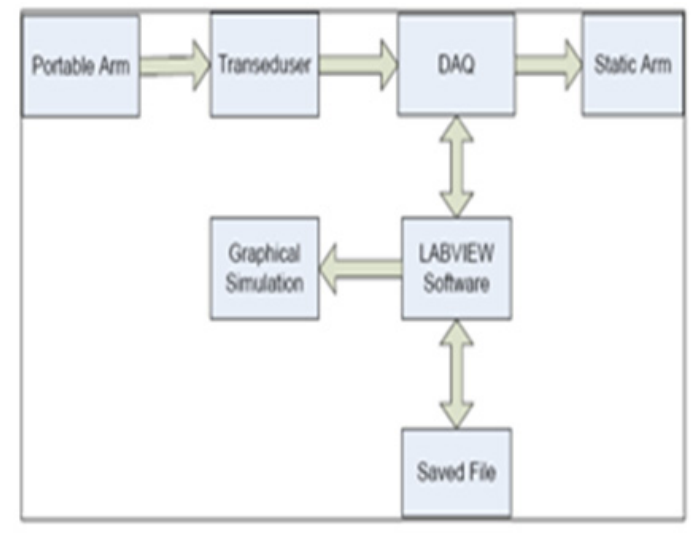

Figure 1. System block diagram

The following subsections explain the arm robot system 
parts in details:

\section{A. Portable arm}

Each of the human arm's joints will be the signal generator for the corresponding joint of the robotic arm's joints. The portable arm shown in Figure (2) is the part responsible for sensing the human arm movements, converting these mechanical signals into electrical signals and then passing them to the controller for processing.

The portable arm consists of four segments with five joints that represent (shoulder, arm, elbow, wrist and gripper) the real human arm. Each of these joints has mechanical-to-electrical signal transducers. The transducer should be chosen to be capable to convert mechanical motion to electrical signal and it is preferable to be rotational according to human's arm joints.

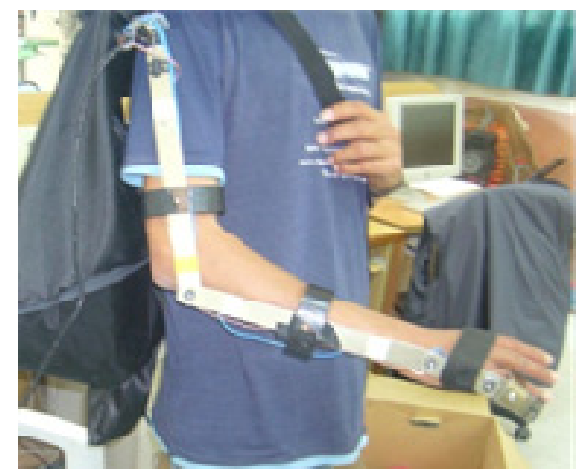

Figure 2. Portable arm

The material used to build the portable arm is aluminum which is preferable to plastic or other materials because it's light weight, ease to fabricate, hard to broke and acceptable appearance.

The potentiometer as shown in Figure (3) is accepted to be the transducer at each joint of the portable arm. Potentiometers are placed on each joint of the arm to measure the rotation. As the joint rotates, the resistance of each sensor varies. This resistance change in turn gives us analog voltages which are fed in to the ADC. Limit switches are placed at all joints to get the extreme positions of the links within the workspace. Limit switches act as over-riding controls for the links. The potentiometer is chosen over other transducer especially optical encoder because it can tell the initial position of the joint at the starting point, it has better resolution than optical encoder, and it has analog input.

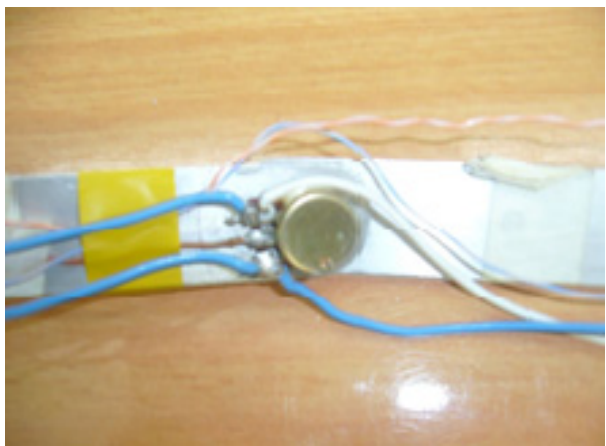

Figure 3. Potentiometer as a transducer

\section{B. Static arm}

The static robot arm consist generally three components, which can perform its task:

- Mechanical arm: As shown in Figure (4), the mechanical design of the robot consists of five parts: the base, shoulder, elbow, wrist and gripper. Plastic is used as the essential material to build the static arm.

- Motors: are used to move the arm to its correct position. In this paper "Servo motor" is used as shown in Figure (5).

- Controller: that processes the input and makes the robot perform its task. LabVIEW, and Data Acquisition Card used as a controller.

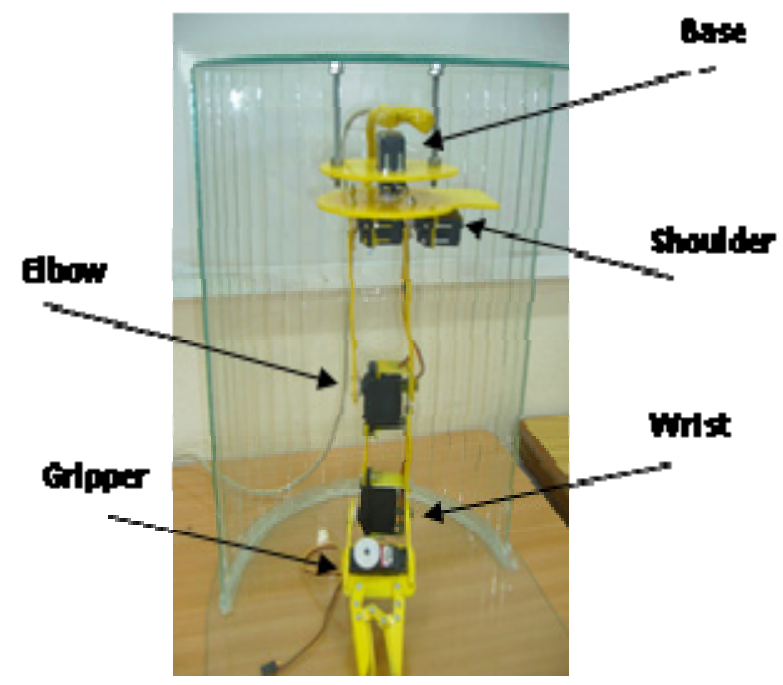

Figure 4. Static Arm

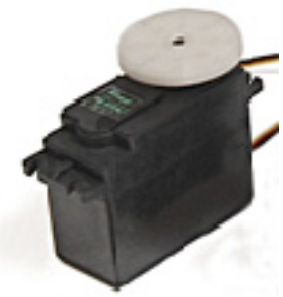

Figure 5. RC Servo Motor

Servo motor

Servo motors (as shown in Figure (5)) were selected as actuators to meet the force/ torque requirements, due to their fast response and less maintenance problems [8]. Five servo motors have been used to drive each of the five joints. Stepper motor or other DC motors can be used to control the arm except that; the DC motor does not have a feedback as servo motor does. Advantages of servo motor over other motors:

1. The stepper motor is controlled by $4-5$ digital bits but the servo used one bit for controlling. As known the DAQ have $8 \mathrm{I} / \mathrm{O}$ digital bit and then can control two stepper motor only but can control 8 servos motor.

2. Most of stepper motors does not have a feedback line.

3. The voltage feedback servo motor is commonly used for position control than encoder (pulse) feedback one. 
4. The cost of the servo is feasible and appropriate.

Each motor can move from 0 to 180 degree and can be modified to move from 0 to 360 degree as shown in Figure (18), but most joint in human arm doesn't need 360 degree rotation. The motors of a robot arm can be best controlled by PWM signals, because the associated power amplifiers will then be able to operate in the pulsed mode which results in minimal power losses.

The advantage of PWM system to produce high voltage and current peaks has been fully utilized to produce larger torques while remaining inside the maximum continuous ratings of the motor. The current surge and voltage spike at startup has been catered for by the PWM control.

\section{Software Control}

The software will be divided into:

\section{Direct Control Mode:}

In this mode the user carries a portable arm which sends the information that represents the rotated angles for each joint to the computer through the data acquisition card (DAQ).

The LabVIEW software program works as a processor that can handle the inputs and interfacing with outputs. As said before the input is represented by the human portable arm and the output will be the static robot arm that simulates the motion of the human arm. In addition, a graphical arm simulation developed beside the static arm.

The program can record a motion with unlimited time by values it captured from the input.

\section{Manual Control Mode:}

In this mode changes has been made on the direct mode that increases the available options of the program and added a new facility of control. The option is focused on controlling the static arm by the program itself, and the inputs in this case will be a numeric angle inputs. As mentioned above the program can record a motion with unlimited time but here the numeric values will play as inputs.

\section{Preview \& Repeat Mode:}

This mode is for browsing a saved motion and can preview the motion or repeats this motion for a specific time. The motion can be break at any time.

The block diagram of the robot arm with the controller is shown in Figure (6).

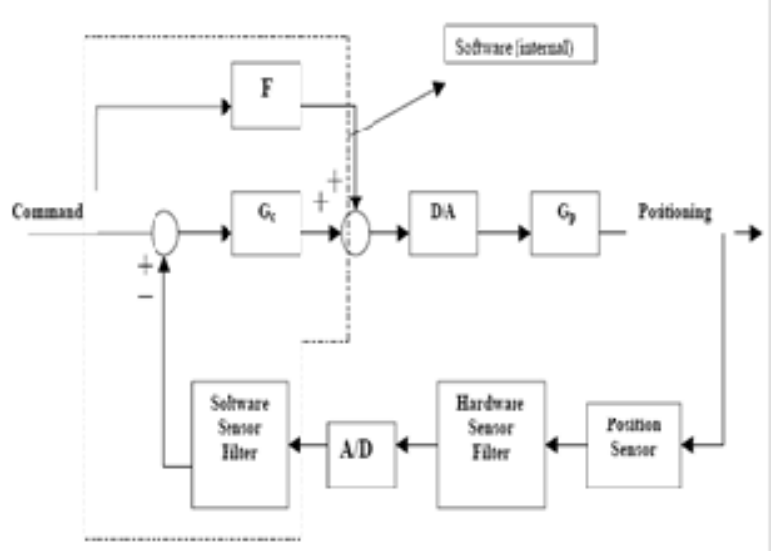

Figure 6. Robot Arm \& Controller Block Diagram

\section{COMPUTER INTERFACING}

Data acquisition system involves gathering signals from measurement sources and digitizing the signal for storage, analysis and presentation on computer. The National Instruments (NI) offers different versions for data acquisition cards: 6023E, 6024E, 6025E. In this paper 6024E shown in Figure (7), has been chosen which features 16 channels of analog input, two channels of analog output, a 68-pin connector and eight lines of digital I/O . This board uses National Instruments DAQ-STC system timing controller for time-related functions. DAQ-STC consists of three timing groups that control analog input, analog output, and general-purpose counter/timer functions. These groups include a total of seven 24-bit and three 16-bit counters and a maximum timing resolution of $50 \mathrm{~ns}$ [9]. DAQ-STC makes possible such applications as buffered pulse generation, equivalent time sampling, and seamless changing of the sampling rate. Three main components to be considered in the systems are [10]:

1) Signals: The signals are standard logic pulses coming from the detectors.

2) DAQ hardware: DAQ hardware acts as the interface between the computer and the outside world.

3) Driver and software

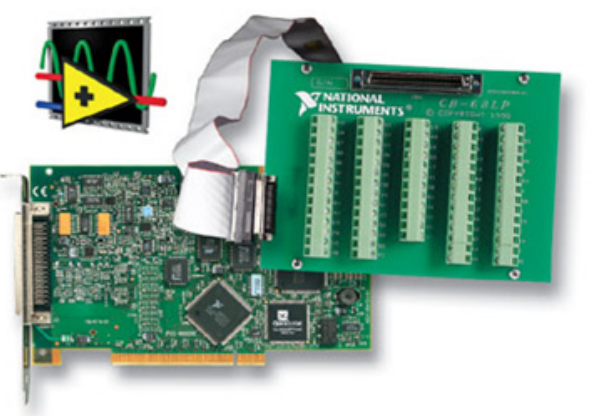

Figure 7. DAQ 6024E and the pin connector

Data acquisition begins with the physical phenomenon to be measured. This physical phenomenon could be the temperature of a room, the intensity of a light source, the pressure inside a chamber, the force applied to an object, or many other things. An effective DAQ system can measure all phenomena. The ability of DAQ system to measure different phenomena depends on the transducer to convert the physical phenomena into signals measurable by the DAQ hardware. Transducers are synonymous with sensors in DAQ systems [11].

\begin{tabular}{|l|l|l|}
\hline Joint & Channel & $\begin{array}{l}\text { Channel pin } \\
\text { number }\end{array}$ \\
\hline Shoulder & ACH0 & 68 \\
\hline Arm & ACH1 & 33 \\
\hline Elbow & ACH2 & 65 \\
\hline Wrist & ACH3 & 30 \\
\hline Gripper & ACH4 & 28 \\
\hline
\end{tabular}

TABLE 1. PORTABLE ARM \& DAQ CONNECTION

\begin{tabular}{|l|l|l|}
\hline Joint & Channel & $\begin{array}{l}\text { Channel pin } \\
\text { number }\end{array}$ \\
\hline Shoulder & DCH0 & 52 \\
\hline Arm & DCH1 & 17 \\
\hline Elbow & DCH2 & 49 \\
\hline
\end{tabular}




\begin{tabular}{|l|l|l|}
\hline Wrist & DCH3 & 47 \\
\hline Gripper & DCH4 & 19 \\
\hline \multicolumn{2}{|c|}{ TABLE 2. STATIC ARM - DAQ INTERFACING } \\
\hline
\end{tabular}

All these features served the arm robotic system to perform its task.

The portable arm has five joints so it will have five signals and these signals need five analog input channels of DAQ. Table 1 shows the name of the joint and its corresponding input channel in DAQ as connected.

The operation of the static arm depends on sending the signals from DAQ to every motor's control line. The static arm connected to DAQ by connecting every control line to a digital output pin in DAQ. Table 2 shows these connections.

\section{LABVIEW SOFTWARE}

LabVIEW, (Laboratory Virtual Instrumentation Engineering Workbench) developed by National Instruments, is a graphical programming environment suited for high level or system level design. It allows integrating different signal processing components or subsystems within a graphical framework. This software package is one of the first graphical programming products, and is currently used in academia and industries for data acquisition, remote control, simulation, and analysis [12]. LabVIEW uses dataflow programming, where the flow of data determines execution. LabVIEW incorporates a graphical user interface (GUI) programming environment to produce programs that mimic laboratory instruments. These programs are called Virtual Instruments (VI).Each VI contains three main parts as shown in Figure (8):

- Front Panel. How the user interacts with the VI.

- Block Diagram. The code that controls the program.

- Icon/Connector. Means of connecting a VI to other VIs.

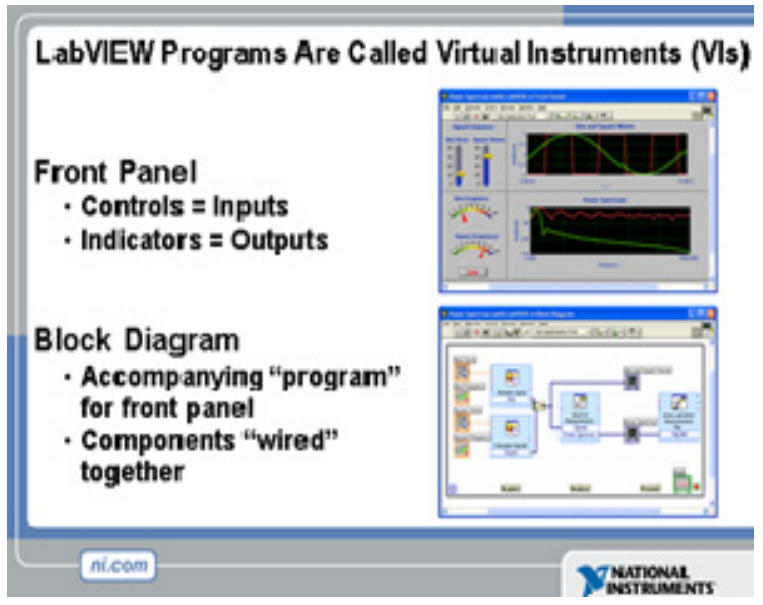

Figure 8. LabVIEW VI

Figure (9) shows the main control panel of the system. The robotic arm system is composed of three parts:
1. Direct motion control
2. Manual motion control
3. Preview \& repeat mode

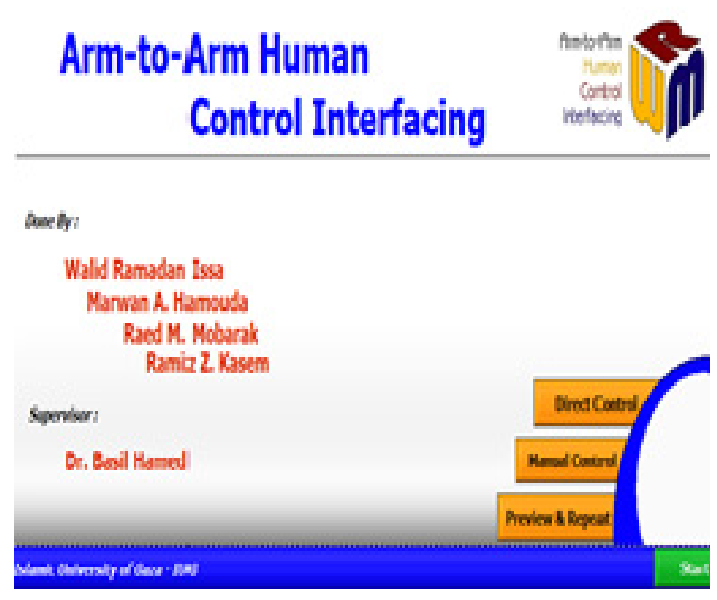

Figure 9. Main Control Panel

1. Direct Control mode enables the user to control the static arm and a graphical simulator arm using the portable arm. The flow chart and LabVIEW of the direct control panel is presented in Figure (10) and Figure (11).

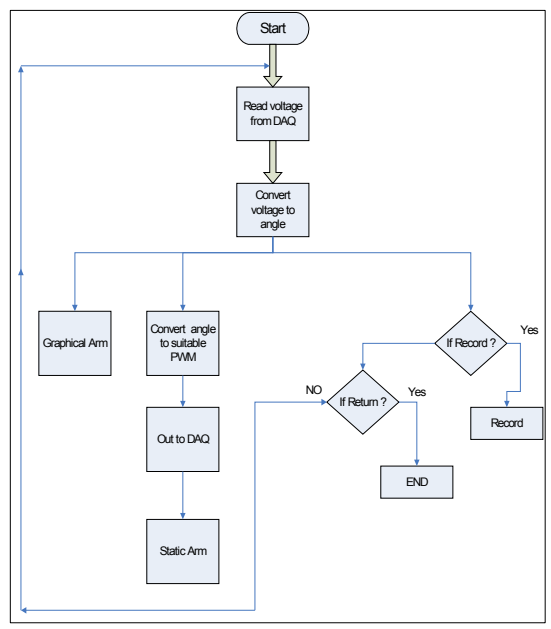

Figure 10. Flow Chart of Direct Control Panel

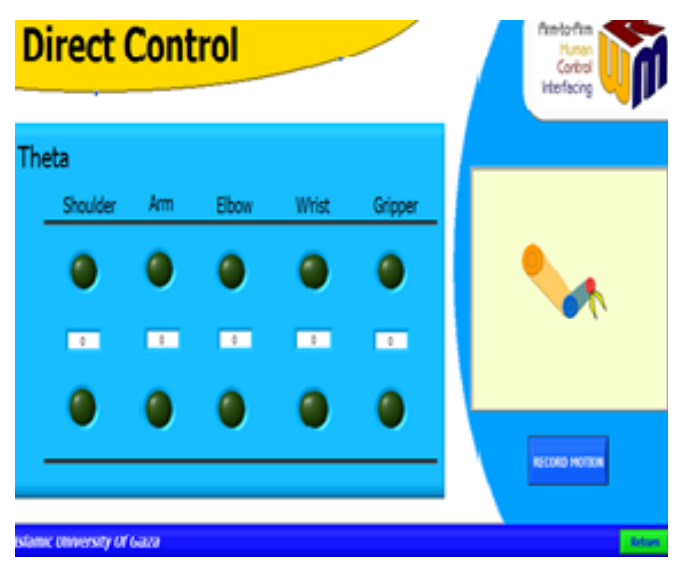

Figure 11. Direct Control Panel

A block diagram of data collected from the portable arm for the direct control mode build using LabVIEW is shown in Figure (12). 


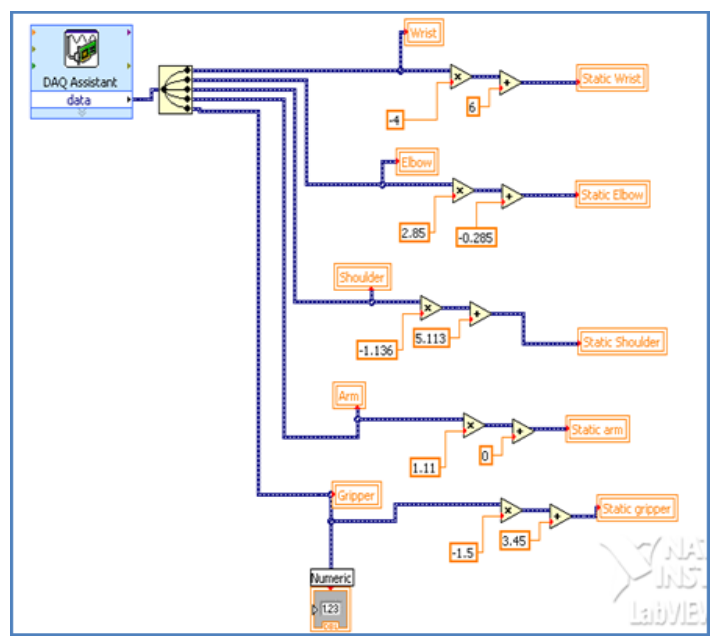

Figure 12. Direct Control Block Diagram

2. Manual Control Panel enables the user to control the static arm from the software itself as shown in Figure (13, and 14)

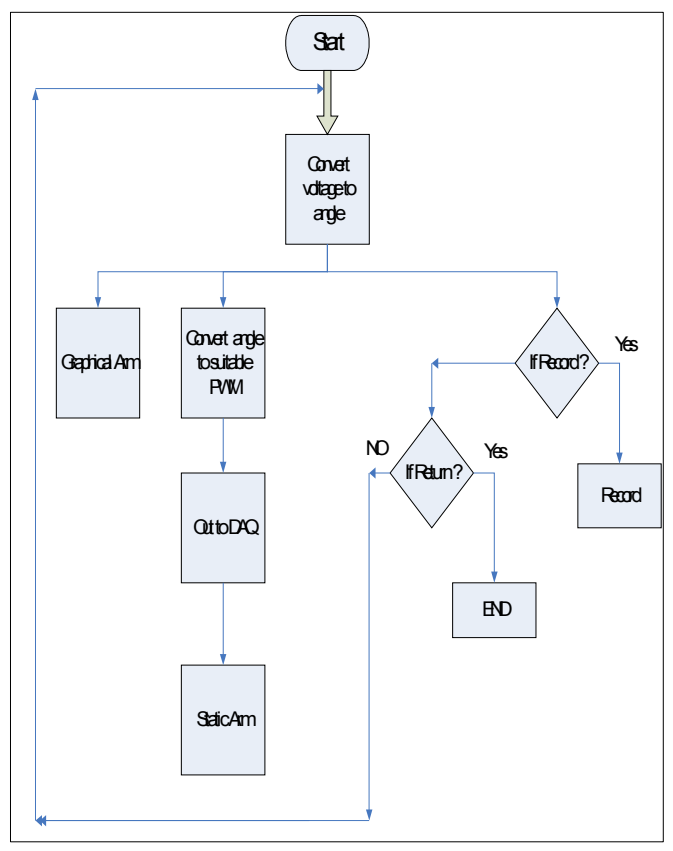

Figure 13. Flow Chart of Manual Control Panel

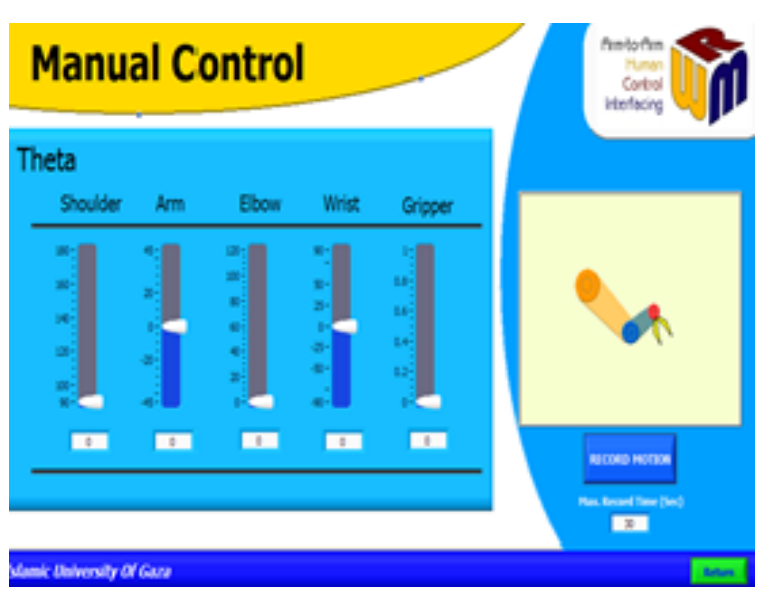

Figure 14. Manual Control Panel
3. Preview \& Repeat Control Panel enables the user to preview the motion recorded in manual or direct panels. If "repeat" is chosen, the Motion is previewed continuously as shown in Figure (15), and Figure (16)

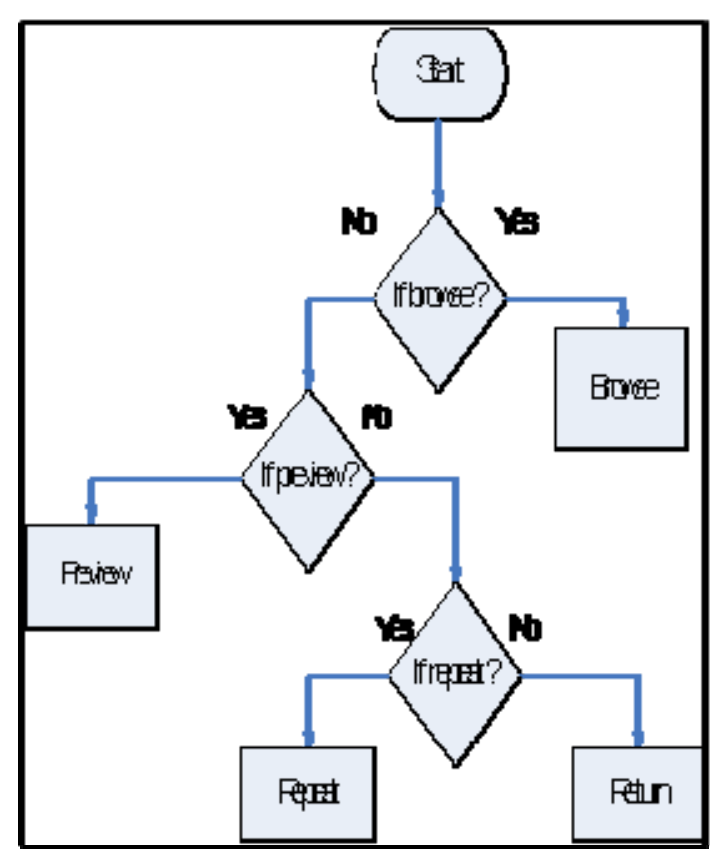

Figure 15. Flow Chart of Preview \& Repeat Control Panel

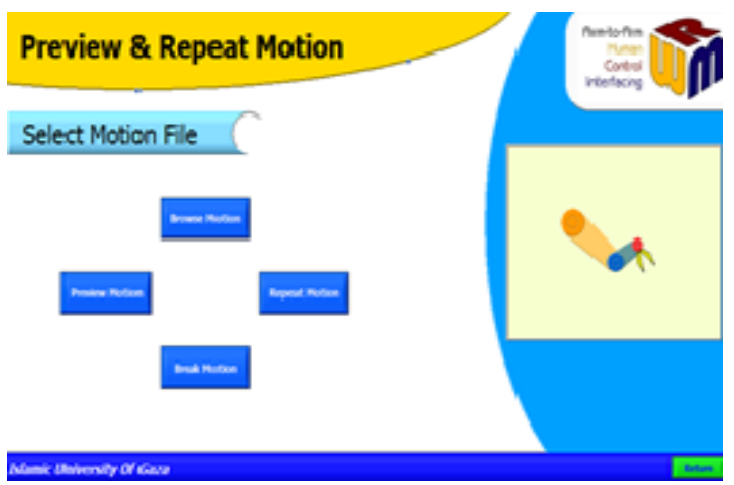

Figure 16. Preview \& Repeat Control Panel

The block diagram of preview \& repeat mode presented in Figure (19) build using LabVIEW

\section{CONCLUSION}

Robot mimicking is an active research area, covering many different fields of science and engineering such as artificial intelligence, interfacing with computer, converting signals (A/D), machine learning, and kinematics. However, when the target to be mimicked is a human arm, intrinsic difficulties for describing the human arm dynamics arise. This paper presents the LabVIEW based data acquisition card system to control the robot arm mimics the human arm. The 5-Jiont mechanical arm which is built from scratch; a hardware robot arm and a portable arm were completely and successfully implemented and linked them using the LabVIEW \& DAQ. The interface developed is powerful tool for controlling the 
robotic arm. Software has been designed in such a way to make it more user-friendly and understandable. It has been tested in many areas and competitions. Its performance was observed to be excellent. Just as it was an educating experience conducting this project, its outcome, can be used as an educational tool for introducing the LabVIEW based data acquisition card real-time applications.

\section{REFERENCES}

[1] Norman S. Nise."Control Systems Engineering", 4th edition. 2004, John Wily \& Sons, Inc

[2] The Canandarm: Remote Manipulator System (RMS), Available:http://ffden-2.phys.uaf.edu/211.web.stuff/Adamczak/rms.ht $\mathrm{m}$

[3] G. Hirzinger, N. Sporer, M. Schedl, J. Butterfass, M. Grebenstein, "Robotics and Mechatronics in aerospace," The 7th International Workshop on Advanced Motion Control, 2002, pp. 19-27.

[4] Y. Woo-Keun, T. Goshozono, H. Kawabe, M. Kinami, Y. Tsumaki, M. Uchiyama, M. Oda, and T. Doi, "Model-based space robot teleoperation of ets-vii manipulator," IEEE Trans. on Robotics and Automation, vol. 20, no. 3, pp. 602-612, 2004.

[5] T. Tayh-Jong, A. Bejczy, G. Chuanfan, and X. Ning, "Intelligent planning and control for telerobotic operations," Proc. of IEEE/RSJ Int Conf. Intelligent Robots and Systems, pp. 389-396, 1994.

[6] J. Park and O. Khatib, "A haptic teleoperation approach based on contact force control," International Journal of Robotics Research, vol. 25 , no. 5-6, pp. 575-591, 2006.

[7] T. Asfour and R. Dillmann, "Human-like motion of a HumanoidRobot Arm Based on a Closed-Form Solution of the InverseKinematics Problem", Industrial Application of Informatics andMicrosystems (IAIM), Computer science Department, University ofKarlsruhe, Germany.

[8] John. J. Craig, "Introduction to Robotics", Mechanics and Control,2nd Edition, Addison-Wesley, 1989.

[9] National Instruments, DAQ 6023E/ 6024E/ 6025E, user manual: January 1999 Edition.

[10] D. F. Styer, The strange world of quantum mechanics $\sim$ cambridge U.P., New York, 2000

[11] http://www.omega.com/prodinfo/data acquisition.html

[12] Korrapati R.; Anderson, J.A; Swain, N.K; Swain M; "System Modeling Using Virtual Instruments" Southeast Con, 2002.Proceedings IEEE Digital Object Identifier:10.1109/ 2002.995570Publication Year: 2002, Page(s): $121-126$
Dr. Basil Hamed is Assistant Professor of Electrical Engineering Department, Islamic University of Gaza, Palestine, since 1999. He has Bachelor Degree in Electrical Engineering from New Mexico State University, NM. USA in the year of 1989, he received Master degree from University of New Orleans, La. USA in the year of 1992, and earned his PhD (Fuzzy Control System) from New Mexico State University, NM USA in the year 1999. He has 15 years of teaching experience and has published many papers in national and international journals. His fields of interest include Control Systems, Fuzzy Control, Simulation \& Modeling, FPGA, Signal and Image Processing

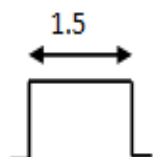

$1.5 \mathrm{~ms}: 90 \mathrm{u}$

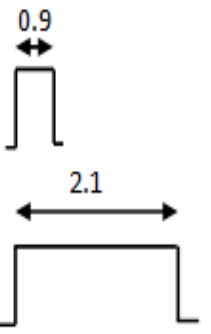

$0.9 \mathrm{~ms}:$

0प्र

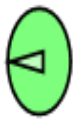

$2.1 \mathrm{~ms}: 180^{\circ}$

1.2

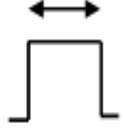

$1.2 \mathrm{~ms}: 45^{\circ}$

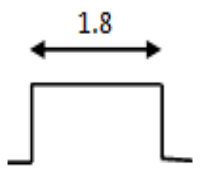

$1.8 \mathrm{~ms}: 135^{\circ}$
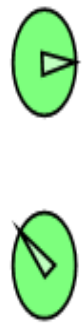

Figure (17) Controlling Servo Position Using Pulse Code Modulation 


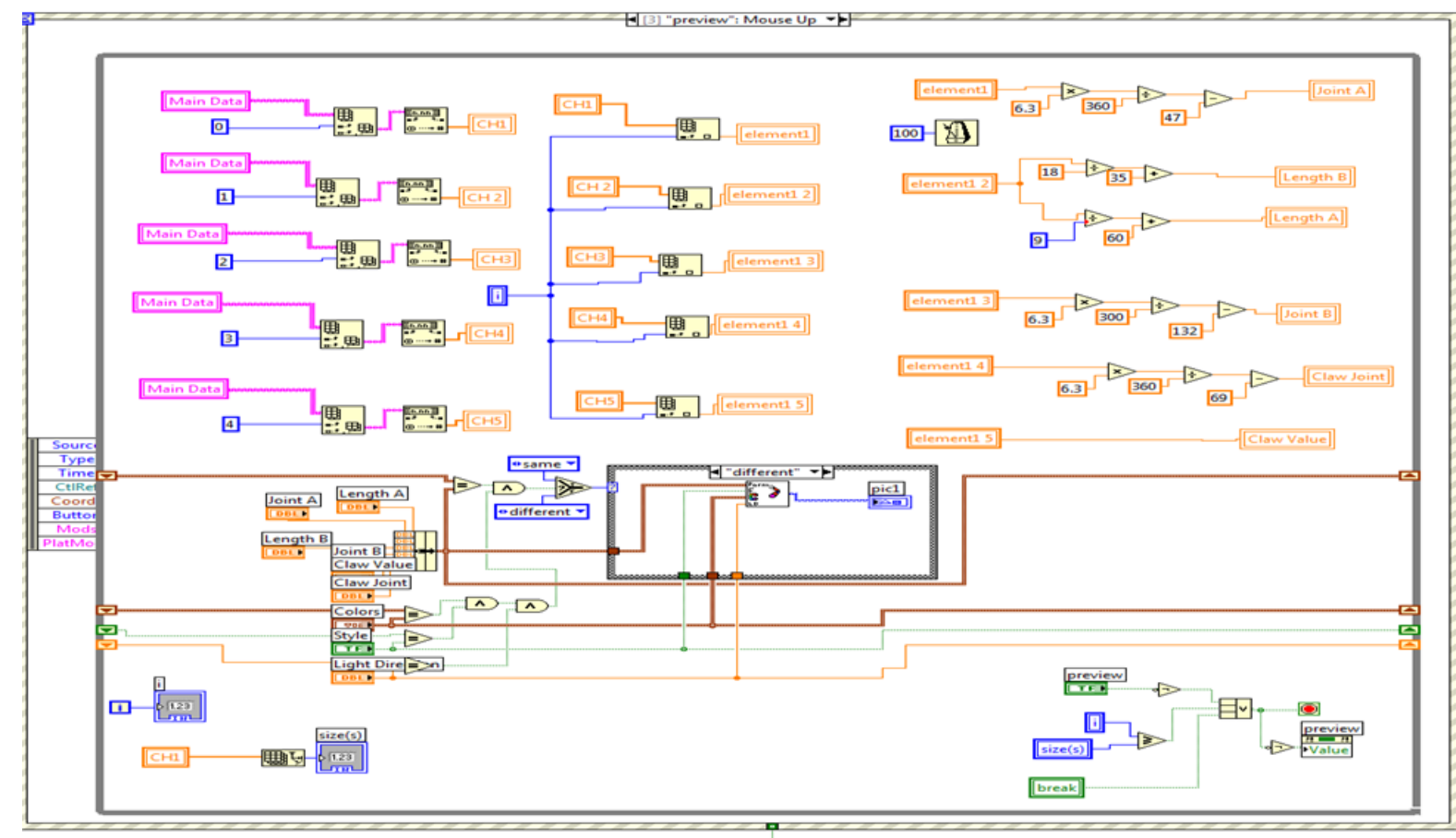

Figure 18. Preview \& Repeat VI Block diagram

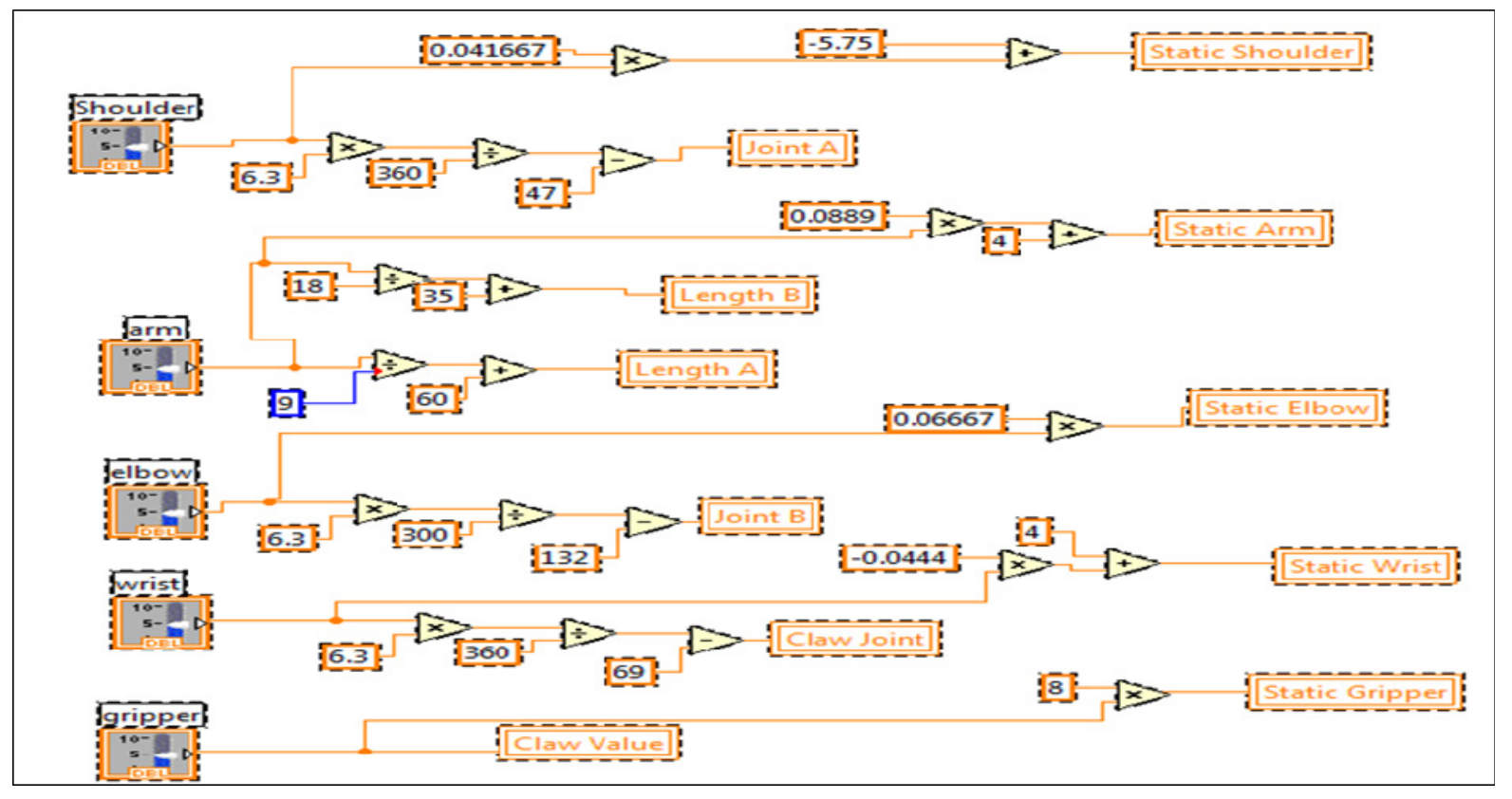

Figure 19. Arm Robot VI Block Diagram 